\title{
Boxes Go Bananas: Encoding Higher-Order Abstract Syntax with Parametric Polymorphism
}

\author{
Geoffrey Washburn Stephanie Weirich \\ Department of Computer and Information Science \\ University of Pennsylvania \\ \{geoffw,sweirich\}@cis.upenn.edu
}

\begin{abstract}
Higher-order abstract syntax is a simple technique for implementing languages with functional programming. Object variables and binders are implemented by variables and binders in the host language. By using this technique, one can avoid implementing common and tricky routines dealing with variables, such as captureavoiding substitution. However, despite the advantages this technique provides, it is not commonly used because it is difficult to write sound elimination forms (such as folds or catamorphisms) for higher-order abstract syntax. To fold over such a datatype, one must either simultaneously define an inverse operation (which may not exist) or show that all functions embedded in the datatype are parametric.

In this paper, we show how first-class polymorphism can be used to guarantee the parametricity of functions embedded in higher-order abstract syntax. With this restriction, we implement a library of iteration operators over data-structures containing functionals. From this implementation, we derive "fusion laws" that functional programmers may use to reason about the iteration operator. Finally, we show how this use of parametric polymorphism corresponds to the Schürmann, Despeyroux and Pfenning method of enforcing parametricity through modal types. We do so by using this library to give a sound and complete encoding of their calculus into System $\mathrm{F}_{\omega}$. This encoding can serve as a starting point for reasoning about higher-order structures in polymorphic languages.
\end{abstract}

\section{Categories and Subject Descriptors}

D.3.3 [PROGRAMMING LANGUAGES]: Language Constructs and Features-abstract data types, polymorphism, control structures; F.3.3 [LOGICS AND MEANINGS OF PROGRAMS]: Software-type structure, program and recursion schemes, functional constructs; F.4.1 [MATHEMATICAL LOGIC AND FORMAL LANGUAGES]: Mathematical Logic_Lambda calculus and related systems, modal logic

Permission to make digital or hard copies of all or part of this work for personal or classroom use is granted without fee provided that copies are not made or distributed for profi t or commercial advantage and that copies bear this notice and the full citation on the fi rst page. To copy otherwise, to republish, to post on servers or to redistribute to lists, requires prior specifi c permission and/or a fee. ICFP'03, August 25-29, 2003, Uppsala, Sweden.

Copyright 2003 ACM 1-58113-756-7/03/0008 ...\$5.00

\section{General Terms}

Languages

\section{Keywords}

Higher-order abstract syntax, modal type system, catamorphism, parametricity, parametric polymorphism

\section{Introduction}

Higher-order abstract syntax (HOAS) is an old and seductively simple technique for implementing a language with functional programming. ${ }^{1}$ The main idea is elegant: instead of representing object variables explicitly, we use metalanguage variables. For example, we might represent the object calculus term $(\lambda x . x)$ with the Haskell expression $\operatorname{lam}(\backslash \mathrm{x} \rightarrow \mathrm{x})$. Doing so eliminates the need to implement a number of tricky routines dealing with object language variables. For example, capture-avoiding substitution is merely function application in the metalanguage. However, outside of a few specialized domains, such as theorem proving, partial evaluation [26], logical frameworks [22] and intensional type analysis [27, 30], higher-order abstract syntax has found limited use as an implementation technique.

One obstacle preventing the widespread use of this technique is the difficulty in using elimination forms, such as catamorphisms ${ }^{2}$, for datatypes containing functions. The general form of catamorphism for these datatypes requires that an inverse be simultaneously defined for every iteration [16]. Unfortunately, many operations that we would like to define with catamorphisms require inverses that do not exist or are expensive to compute.

However, if we know that the embedded functions in a datatype are parametric, we can use a version of the catamorphism that does not require an inverse $[9,24]$. A parametric function may not examine its argument; it may only use it abstractly or "push it around". Only allowing parametric embedded functions works well with HOAS because the terms with non-parametric embedded functions are exactly those that have no correspondence to any $\lambda$-calculus term [24]. In this paper, we use iterator to refer to a catamorphism restricted to arguments with parametric functions.

A type system can separate parametric functions from those that

\footnotetext{
${ }^{1}$ While the name comes from Pfenning and Elliott [21], the idea itself goes back to Church. [4].

${ }^{2}$ Catamorphisms (also called folds) are sometimes represented with the bananas $(|\cdot|)$ notation [15].
} 
are not. For example, Fegaras and Sheard [9] add tags to mark the types of datatypes whose embedded functions are not parametric, prohibiting iteration over those datatypes. Alternatively, Schürmann, Despeyroux and Pfenning [24, 8] use the necessity modality ("box") to mark those terms that allow iteration.

However, many modern typed languages already have a mechanism to enforce that an argument be used abstractly-parametric polymorphism. It seems desirable to find a way to use this mechanism instead of adding a separate facility to the type system. In this paper, we show how to encode datatypes with parametric function spaces in the polymorphic $\lambda$-calculus, including iteration operators over them.

Our specific contributions are the following. For functional programmers, we provide an informal description of how restricting datatypes to parametric function spaces can be enforced in the Haskell language using first-class polymorphism. We provide a safe and easy implementation of a library for iteration over higher-order abstract syntax. This Haskell library allows the natural expression of many algorithms over the object language; to illustrate its use, we use it to implement a number of operations including Danvy and Filinski's optimizing one-pass CPS conversion algorithm [6]. Furthermore, because we encode the iteration operator within the polymorphic $\lambda$-calculus, we also derive "fusion laws" about the iteration operator that functional programmers may use to reason about their programs.

To show the generality of this technique, we use this implementation to show a formal translation from the Schürmann, Despeyroux and Pfenning modal calculus [24] (called here the SDP calculus) to System $\mathrm{F}_{\omega}$. This encoding has an added benefit to language designers who wish to incorporate reasoning about parametric function spaces. It demonstrates how systems based on the polymorphic $\lambda$-calculus may be extended with reasoning about higher-order structure.

We do not claim that this encoding will solve all of the problems with programming using higher-order abstract syntax. In particular, algorithms that require the explicit manipulation of the names of bound variables remain outside the scope of this implementation technique.

The remainder of this paper is as follows. Section 2 starts with background material on catamorphisms for HOAS, including those developed by Meijer and Hutton [16] and Fegaras and Sheard [9]. In Section 2.1 we show how to use first-class polymorphism and abstract types to provide an interface for Fegaras and Sheard's implementation that enforces the parametricity of embedded functions. Using this interface, we show some examples of iteration including CPS conversion (Section 2.2). In Section 3, we describe an implementation of that interface within the part of Haskell that corresponds to System $\mathrm{F}_{\omega}$, and describe properties of that implementation in Section 3.1. Section 4 describes the SDP calculus and Section 5 presents an encoding of that calculus into $\mathrm{F}_{\omega}$, using the implementation that we developed in Section 3. Section 6 presents future work, Section 7 presents related work, and Section 8 concludes. We include Generic Haskell code for the polytypic part of our implementation in Appendix A and the full encoding of the SDP calculus into System $\mathrm{F}_{\omega}$ in Appendix B.

\section{Catamorphisms for datatypes with embed- ded functions}

The following recursive datatype represents the untyped $\lambda$-calculus using Higher-Order Abstract Syntax (HOAS). ${ }^{3}$

data $\operatorname{Exp}=$ Lam $(\operatorname{Exp} \rightarrow \operatorname{Exp}) \mid$ App Exp Exp

The data constructor Lam represents $\lambda$-expressions. However, instead of explicitly representing bound $\lambda$-calculus variables, Haskell functions are used to implement binding and Haskell variables are used to represent variables. For example, we might represent the identity function $(\lambda x . x)$ as Lam $(\backslash \mathrm{x} \rightarrow \mathrm{x})$ or the infinite loop $(\lambda x .(x x))(\lambda x .(x x))$ as $\operatorname{App}(\operatorname{Lam}(\backslash \mathrm{x} \rightarrow \operatorname{App} \mathrm{x} \mathrm{x}))(\operatorname{Lam}(\backslash \mathrm{x} \rightarrow \operatorname{App} \mathrm{x} x))$.

Using this datatype, we can implement an interpreter for the $\lambda$ calculus. To do so, we must also represent the result values (also using HOAS).

data Value $=$ Fn (Value $\rightarrow$ Value)

unFn $(F n x)=x$

It is tricky to define recursive operations, such as evaluation, over this implementation of expressions. The argument, $\mathrm{x}$, to Lam below is a function of type Exp $\rightarrow$ Exp. To evaluate it, we must convert $x$ to a function of type Value $\rightarrow$ Value. Therefore, we must also simultaneously define an inverse to evaluation, called uneval, such that eval . uneval $=\backslash \mathrm{x} \rightarrow \mathrm{x}$. This inverse is used to convert the argument of $\mathrm{x}$ from a Value to an Exp.

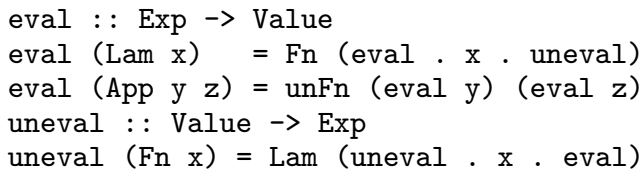

Consider the evaluation of $((\lambda x . x)(\lambda y \cdot y))$. First eval replaces App with unFn and pushes evaluation down to the two subcomponents of the application. Next, each Lam is replaced by Fn, and the argument is composed with eval and uneval. The unFn cancels the first Fn, and the identity functions can be removed from the compositions. As uneval is right inverse to eval, we can replace each (eval . uneval) with the identity function.

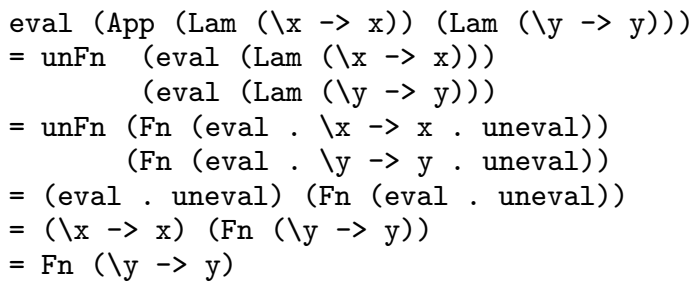

Many functions defined over Exp will follow this same pattern of recursion, requiring an inverse for Lam and calling themselves recursively for the subcomponents of App. Catamorphisms capture the general pattern of recursion for functions defined over recursive datatypes. For example, foldl is a catamorphism for the list datatype and can implement many list operations. For lists of type

\footnotetext{
${ }^{3}$ All of the following examples are in the syntax of the Haskell language [19]. While some of the later examples require an extension of the Haskell type system-first-class polymorphism-this extension is supported by the Haskell implementations GHC and Hugs.
} 


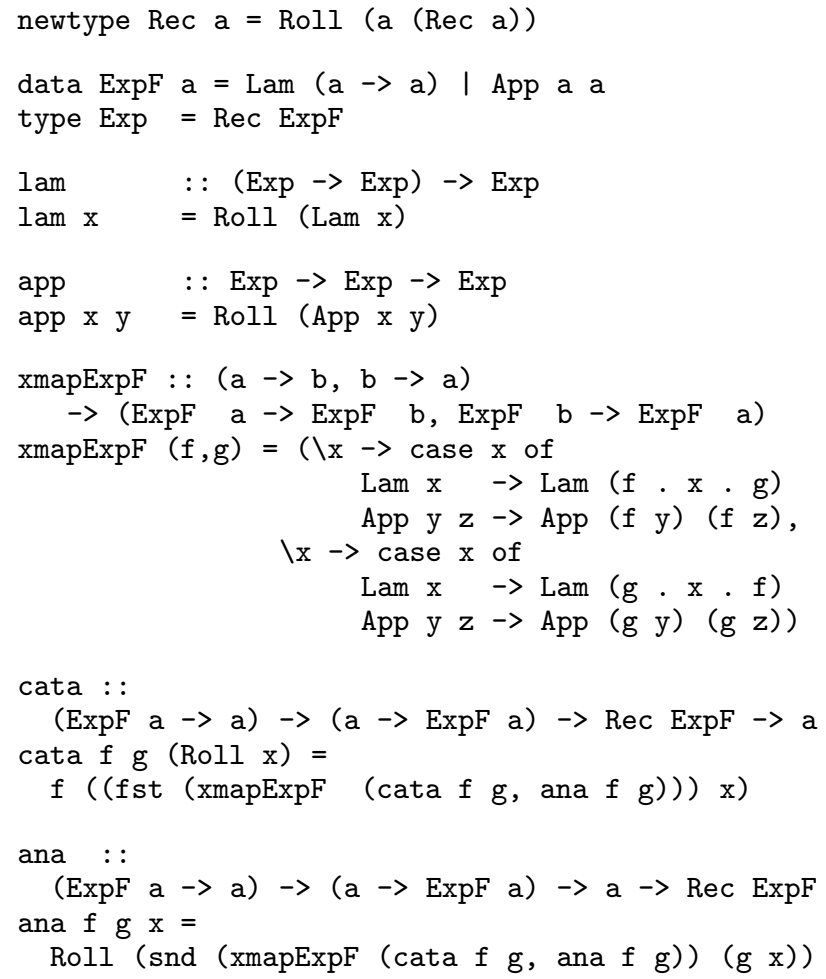

Figure 1. Meijer/Hutton catamorphism

[a], foldr replaces [] with a base case of type b and (: ) with a function of type $(a \rightarrow b \rightarrow b)$.

Meijer and Hutton [16] showed how to define catamorphisms for datatypes with embedded functions, such as Exp. The catamorphism for Exp systematically replaces Lam with a function of type $((a->a) \rightarrow a)$ and App with a function of type $(\mathrm{a} \rightarrow \mathrm{a} \rightarrow \mathrm{a})$. However, just as we defined eval simultaneously with uneval, the catamorphism for Exp must be simultaneously defined with an anamorphism. The catamorphism provides a way to consume members of type Exp and the anamorphism provides a way to generate them.

In order to easily specify this anamorphism, we use a slightly more complicated version of the Exp datatype, shown at the top of Figure 1. This version makes the recursion in the datatype explicit. The newtype Rec computes the fixed point of type constructors (functions from types to types). The type $\operatorname{Exp}$ is the fixed point of the type constructor ExpF, where the recursive occurrences of Exp have been replaced with the type parameter $a$. The first argument to cata is of type $\operatorname{ExpF} a \rightarrow$ a (combining the two functions mentioned above, of type $((a->a) \rightarrow a)$ and $(a->a \rightarrow a))$. The first argument to ana has the inverse type a $\rightarrow \operatorname{ExpF}$ a.

The functions cata and ana are defined in terms of xmapExpF, a generalized version of a mapping function for the type constructor $\operatorname{ExpF}$. Because of the function argument to Lam, xmapExpF maps two functions, one of type $a->b$ and the other of type $\mathrm{b} \rightarrow \mathrm{a}$. The definition of xmapExpF is completely determined by the definition of ExpF. With Generic Haskell [5], we can define xmap and automatically generate xmapExpF from ExpF (see Ap-

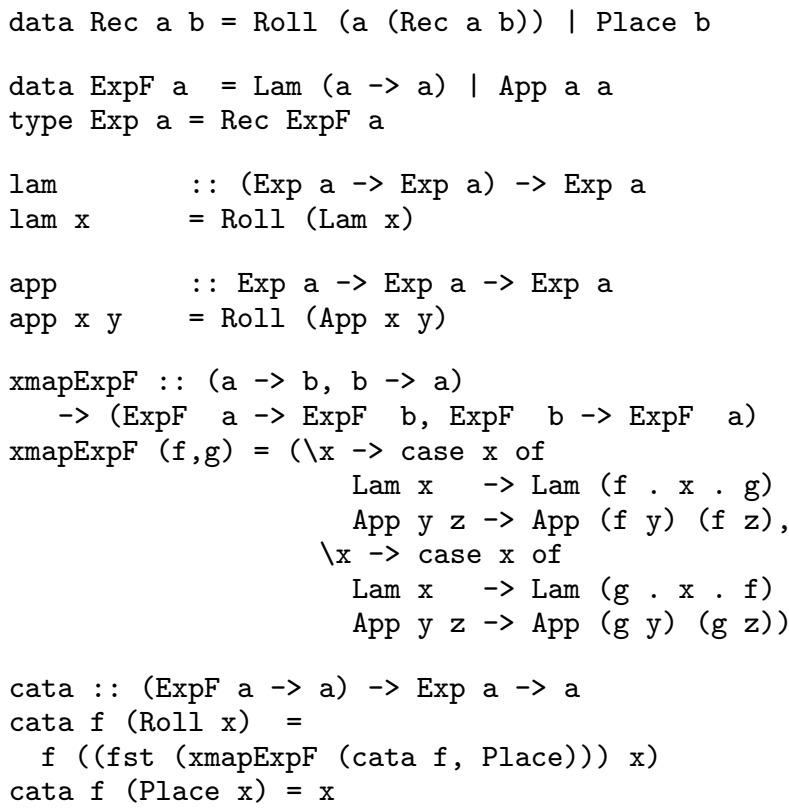

Figure 2. Fegaras/Sheard catamorphism

pendix A). ${ }^{4}$ That way, we can easily generalize this catamorphism to other datatypes. Unlike map, which is defined only for covariant type constructors, xmap is defined for type constructors that have both positive and negative occurrences of the bound variable. The only type constructors of $\mathrm{F}_{\omega}$ for which xmap is not defined are those whose bodies contain first-class polymorphism. For example, $\lambda \alpha: \star . \forall \beta: \star . \alpha \rightarrow \beta$.

We can use cata to implement eval. To do so we must describe one step of turning an expression into a value (the function evalAux) and one step of turning a value into an expression (the function unevalAux).

evalAux : : ExpF Value $\rightarrow$ Value

evalAux (Lam $f$ ) $=F n f$

evalAux (App $x$ y) $=(u n F n x) y$

unevalAux :: Value $\rightarrow$ ExpF Value

unevalAux $(F n f)=\operatorname{Lam} f$

eval : : Exp $\rightarrow>$ Value

eval $\mathrm{x}=$ cata evalAux unevalAux $\mathrm{x}$

Using cata to implement operations such as eval is convenient because the pattern of recursion is already specified. None of eval, evalAux or unevalAux are recursively defined. However, for some operations, there is no obvious (or efficient) inverse. For example, to using cata to print out expressions also requires writing a parser. Fegaras and Sheard [9] noted that sometimes the operation of the catamorphism often undoes with $f$ what it has just done with

\footnotetext{
${ }^{4}$ Meijer and Hutton's version of xmapExpF only created the first component of the pair. In ana where the second component is needed, they swap the arguments. This is valid because $f$ st (xmap $(f, g))=\operatorname{snd}(\operatorname{xmap}(g, f))$. However, while the version that we use here is a little more complicated, it can be defined with Generic Haskell.
} 
g. This situation occurs when the argument to cata contains only parametric functions. A parametric function is one that does not analyze its argument with case or cata.

When the argument to cata is parametric, Fegaras and Sheard showed how to implement cata without ana. The basic idea is that for parametric functions, any use of ana during the computation of a catamorphism will always be annihilated by cata in the final result. Therefore, instead of computing the anamorphism, they use a place holder to store the original argument. When cata reaches that place holder, it returns the stored argument.

To implement Fegaras and Sheard's catamorphism, we must redefine Rec. In Figure 2, we extend it with an extra branch (called Place) that is the place holder. Because Place can contain any type of value, Rec (and consequently Exp) must be parameterized with the type of the argument to Place. This type is the result of the catamorphism over the expression. In the implementation of cata, Place is the second argument to xmapExpF instead of ana $f$. It is a right inverse to cata $f$ by definition.

For example, to count the number of occurrences of bound variables in an expression, we might use the following code.

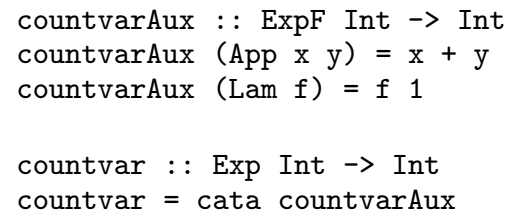

The function countvarAux describes what to do in one step. The number of variables in an application expression is the sum of the number of variables in $\mathrm{x}$ and the number of variables in $\mathrm{y}$. In the case of a $\lambda$-expression, $f$ is a function from the number of variables in a variable expression (i.e. one) to the number of variables in the body of the lam. For example, to count the variables in $(\lambda x . x x)$ :

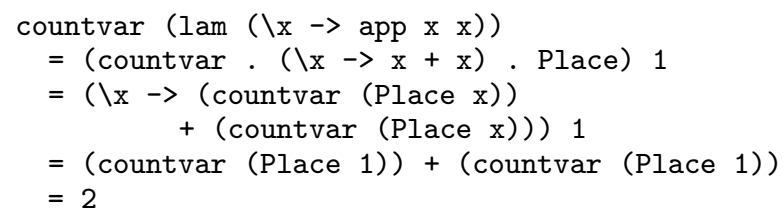

This definition of cata only works for arguments whose function spaces are parametric and who do not use Place. Informally, we call such expressions sound and other expressions unsound. Applying cata to an unsound expression can return a meaningless result. For example, say we define the following term:

badplace : : Exp Int

badplace $=\operatorname{lam}(\backslash \mathrm{x} \rightarrow$ Place 3$)$

Then countvar badplace $=3$, even though it contains no bound variables. Even more importantly for higher-order abstract syntax, unsound datatypes do not correspond to untyped $\lambda$-calculus expressions, so it is important to be able to distinguish between sound and unsound representations. ${ }^{5}$

\footnotetext{
${ }^{5}$ It is also important to distinguish between sound and unsound members of datatypes that have meaningful non-parametric representations. For these datatypes, the behavior of the Fegaras and Sheard catamorphism on unsound arguments does not correspond to the Meijer and Hutton version.
}

There are two ways for parametricity to fail, corresponding to the two destructors for the type $\operatorname{Exp}$ a. A function is not parametric if it uses cata or case to examine its argument, as below:

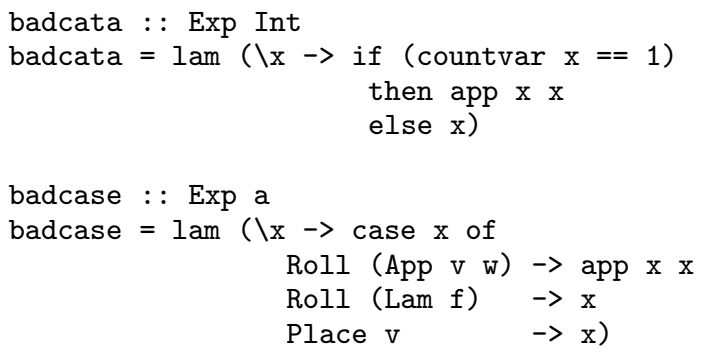

Fegaras and Sheard designed a type system to distinguish between sound and unsound expressions. Datatypes such as Exp were annotated with flags to indicate whether they had been examined with either case or cata, and if so, they were prevented from appearing inside of non-flagged datatypes. Furthermore, their language prevented the user from accessing Place by automatically generating cata from the definition of the user's datatype.

\subsection{Enforcing parametricity with type ab- straction}

The type of badcata is Exp Int. This type tells us that something is wrong: the type parameter of Exp is constrained to be Int, so we can only use cata on this expression to produce an Int. The same is true for badplace. Whenever we use cata or Place in an expression, this parameter will be constrained. If we can ensure that only sound expressions have type (forall a. Exp a), then we can use first-class polymorphism to enforce that the argument to a function is sound. That way, we can be assured that it will behave as expected. For example, define a version of cata, called iter 0 that may only be applied to sound expressions, below. The implementation of cata uses the argument at the specific type (Exp a), so it is safe for iter0 to require that its argument has the more general type (forall a. Exp a).

iter0 : : (ExpF b $\rightarrow$ b) $\rightarrow($ forall a. $\operatorname{Exp} a) \rightarrow b$

iter0 $=$ cata

However, this new type does not prevent expressions like badcase from being the argument to iter0. We can prevent such case analysis inside lam expressions by ruling out case analysis for all terms of type $\operatorname{Exp} t$. If the user cannot use case, then they cannot write badcase. While this restriction means that some operations cannot be naturally defined in this calculus, cata alone can define a large number of operations, as we demonstrate below and in Section 2.2.

There are two ways to prohibit case analysis. The first way is to reimplement Exp in such a way that cata is the only possible operation (in other words without using a Haskell datatype). We discuss this alternative in Section 3.

The second way to prohibit case analysis is to make Rec an abstract type constructor. If the definition of Rec is hidden by some module boundary, such as with the interface in Figure 3, then the only way to destruct an expression of type $\operatorname{Exp}$ a is with cata. Because Roll and Place are datatype constructors of Rec, and cata pattern matches these constructors, they must all be defined in the same module as Rec. However, because we only need to prohibit case analysis, we can export Roll and Place as the functions roll and place. With roll we can define the terms app and lam anywhere. 


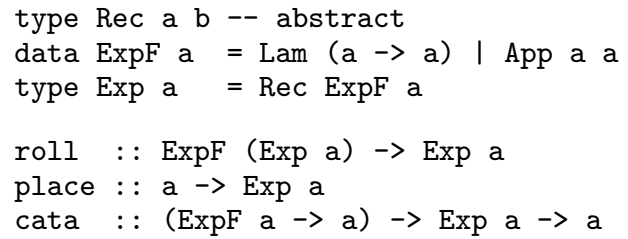

\section{Figure 3. Iteration library interface}

We can also make good use of place. The type forall a. Exp a enforces that all embedded functions are parametric, but it can only represent closed expressions. What if we would like to examine expressions with free variables? In HOAS, an expression with one free variable has type $\operatorname{Exp} t \rightarrow \operatorname{Exp} t$. To compute the catamorphism for the expression, we use place to provide the value for the free variable.

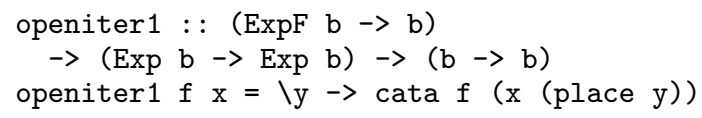

If we would like to make sure that the expression is sound, we must quantify over the parameter type and require that the expression have type forall a. Exp a $\rightarrow \operatorname{Exp}$ a.

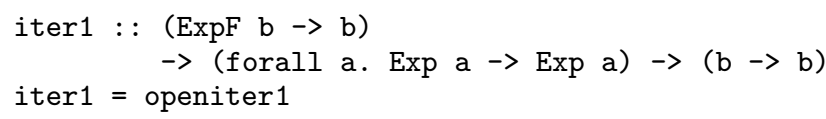

With iter 1 we can determine if that one free variable occurs in an expression.

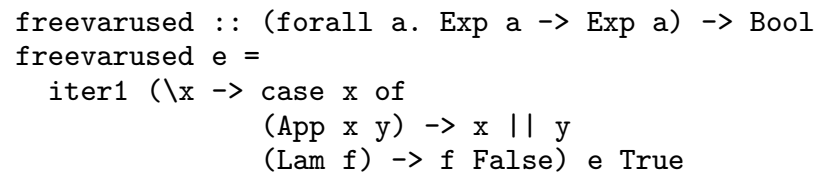

An app expression uses the free variable if either the function or the argument uses it. The occurrence of the bound variable of a lam is not an occurrence of the free variable, so False is the argument to $f$, but the expression does use the free variable if it appears somewhere in the body of the abstraction. Finally, the program works by feeding in True for the value of the free variable. If the result is True then it must have appeared somewhere in the expression.

There is no reason to stop with one free variable. There are an infinite number of related iteration operators, each indexed by the type inside the forall. The types of several such iterators are shown below. For example, the third one, iterList, may analyze expressions with arbitrary numbers of free variables.

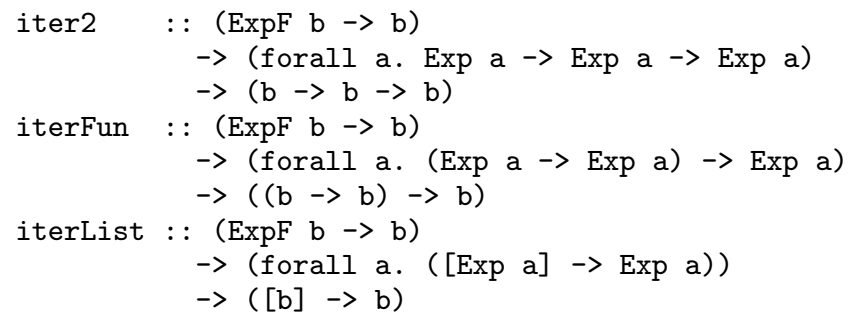

Each of these iterators is defined by using xmap to map (cata $f$ ) and place. Thus we can easily implement them by defining the appropriate version of xmap. However, because xmap is a polytypic function, we should be able to automatically generate all of these iterators using Generic Haskell. The following code implements these operations. Below, the notation $\operatorname{xmap}\{|\mathrm{g}|\}$ generates the instance of xmap for the type constructor $g$.

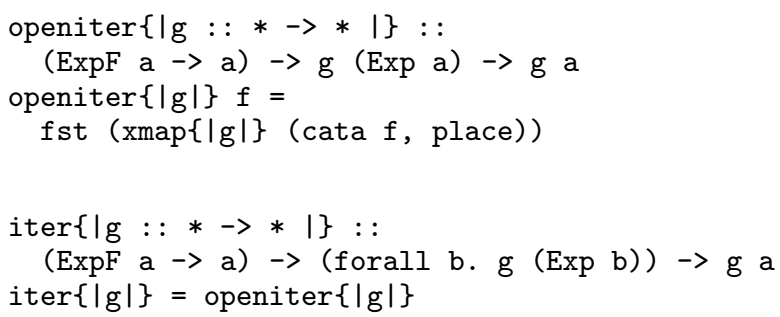

Unfortunately, the above Generic Haskell code cannot automatically generate all the iterators that we want, such as iter1, iterFun and iterList. Because of type inference, $g$ can only be a type constructor that is a constant or a constant applied to type constructors [13]. In particular, we cannot represent the type constructor $(\lambda \alpha: \star . \alpha \rightarrow \alpha)$ in Haskell, so we cannot automatically generate the instance

$$
\begin{aligned}
& \text { iter1 : : ( } f \quad b->b) \\
& \rightarrow(\text { forall a. }(\operatorname{Exp} a) \rightarrow(\operatorname{Exp} a)) \rightarrow b \rightarrow b
\end{aligned}
$$

Fortunately, using a different extension of Haskell, called functional dependencies [14], we can generate these versions of openiter. For each version of iter that we want, we still need to redefine the generated openiter with the more restrictive type.

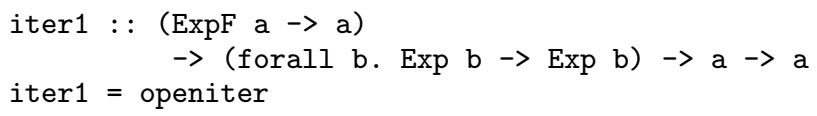

The Iterable class defines openiter simultaneously with its inverse. The parameters $m$ and $n$ should be $g(\operatorname{Exp} a)$ and $g$ a, where each instance specifies $g$. (The type $a$ is a parameter of the type class so that $m$ and $n$ may refer to it.) Also necessary are the functional dependencies that state that $m$ determines both a and $n$. These dependencies rule out ambiguities during type inference.

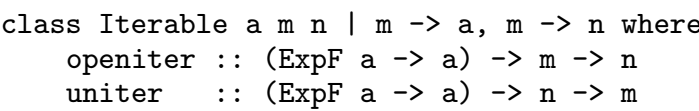

If $g$ is the identity type constructor, then $m$ and $n$ are Exp a and a respectively.

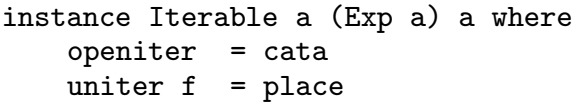

Using the instances for the subcomponents, we can define instances for types that contain $\rightarrow$.

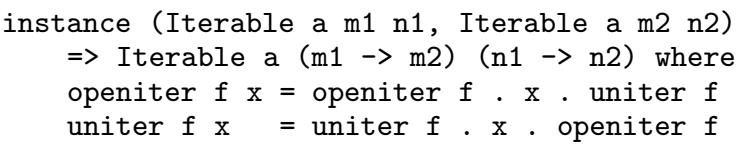

With these instances, we have a definition for openiter $\{\mid \lambda \alpha . \alpha \rightarrow$ $\alpha \mid$ \}. It is not difficult to add instances for other type constructors, such as lists and tuples. 


\subsection{Examples of iteration}

We next present several additional examples of the expressiveness of iter0 for arguments of type (forall a. Exp a). The purpose of these examples is to demonstrate how to implement some of the common operations for $\lambda$-calculus terms without case analysis.

For example, we can use iter0 to convert expressions to strings. So that we have different names for each nested binding occurrence, we must parameterize this iteration with a list of variable names. Haskell's list comprehension provides us with an infinite supply of strings.

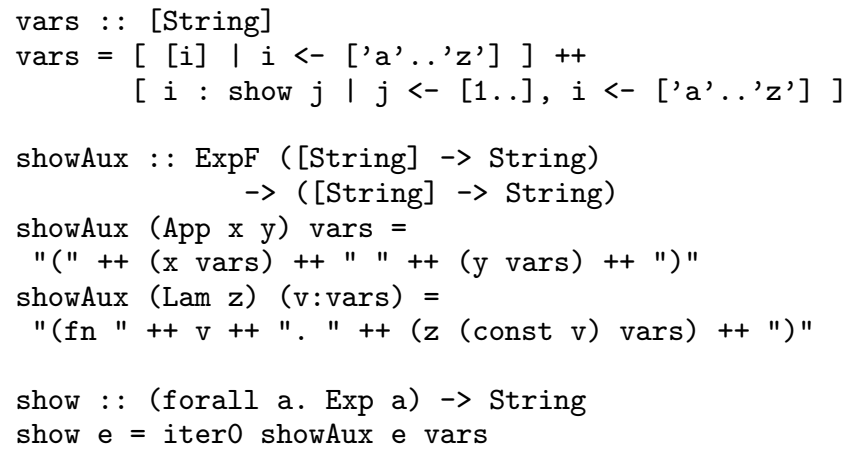

Applying show to an expression produces a readable form of the expression.

show $(\operatorname{lam}(\backslash x \rightarrow \operatorname{lam}(\backslash y \rightarrow \operatorname{app} x$ y))

$=(f n a \cdot(f n b \cdot(a b)))$

Another operation we might wish to perform for a $\lambda$-calculus expression is to reduce it to a simpler form. As an example, we next implement parallel reduction for a $\lambda$-calculus expression. ${ }^{6}$ Parallel reduction differs from full reduction in that it does not reduce any newly created redexes. Therefore, it terminates even for expressions with no $\beta$-normal form. Parallel reduction may be specified by the following inductive definition.

$$
\begin{gathered}
\overline{x \Rightarrow x} \quad \frac{M \Rightarrow M^{\prime}}{\lambda x \cdot M \Rightarrow \lambda x \cdot M^{\prime}} \quad \frac{M \Rightarrow M^{\prime} \quad N \Rightarrow N^{\prime}}{M N \Rightarrow M^{\prime} N^{\prime}} \\
\frac{M \Rightarrow M^{\prime} \quad N \Rightarrow N^{\prime}}{(\lambda x \cdot M) N \Rightarrow M^{\prime}\left\{x / N^{\prime}\right\}}
\end{gathered}
$$

We use iter0 to implement parallel reduction below. The tricky part is the case for applications. We must determine whether the first component of an application is a lam expression, and if so, perform the reduction. However, we cannot do a case analysis on expressions, as the type Exp a is abstract. Therefore, we implement parallel reduction with a "pairing" trick ${ }^{7}$. As we iterate over the term we produce two results, stored in the following record:

$$
\begin{aligned}
& \text { data PAR a }=\text { PAR }\{\text { par : : Exp a, } \\
& \text { apply :: Exp a } \rightarrow \text { Exp a }\}
\end{aligned}
$$

The first component, par, is the actual result we want - the parallel reduction of the term. The second component, apply, is a func-

\footnotetext{
${ }^{6}$ This example is from Schürmann et. al [24].

${ }^{7}$ Pairing was first used to implement the predecessor operation for Church numbers. The iteration simultaneously computes the desired result with auxiliary operations.
}

tion that we build up for the application case. In the case of a lam expression, apply performs the substitution in the reduced term. Otherwise, apply creates an app expression with its argument and the reduced term. 8

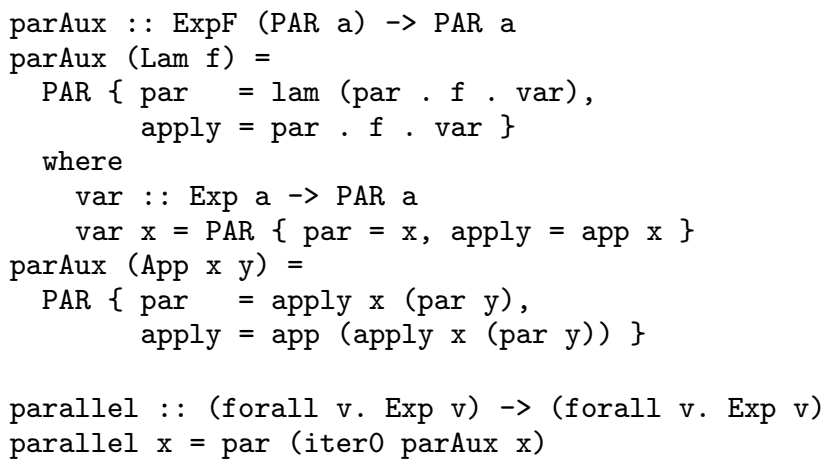

For example:

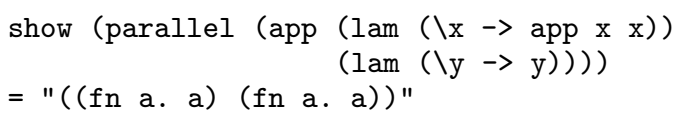

While we could not write the most natural form of parallel reduction with iter0, other operations may be expressed in a very natural manner. For example, we can implement the one-pass call-byvalue CPS-conversion of Danvy and Filinski [6]. This sophisticated algorithm performs "administrative" redexes at the meta-level so that the result term has no more redexes than the original expression. The algorithm is based on two mutually recursive operations: cpsmeta performs closure conversion given a meta-level continuation (a term of type Exp a $\rightarrow$ Exp a), and cpsobj does the same with an object-level continuation (a term of type Exp a).

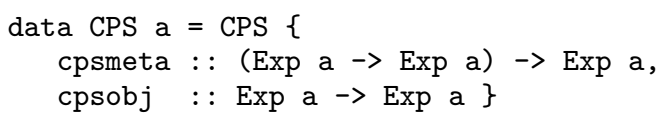

If we are given a value (i.e. a $\lambda$-expression or a variable) the function value below describes its CPS conversion. Given a metacontinuation $\mathrm{k}$, we apply $\mathrm{k}$ to the value. Otherwise, given an object continuation $c$, we create an object application of $c$ to the value.

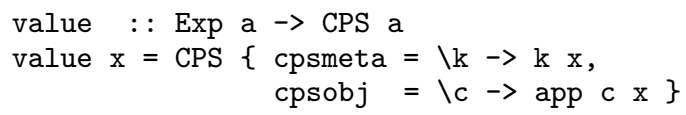

The operation cpsAux takes an expression whose subcomponents have already been CPS converted and CPS converts it. For application, translation is the same in both cases except that the meta-case converts the meta-continuation into an object continuation with lam.

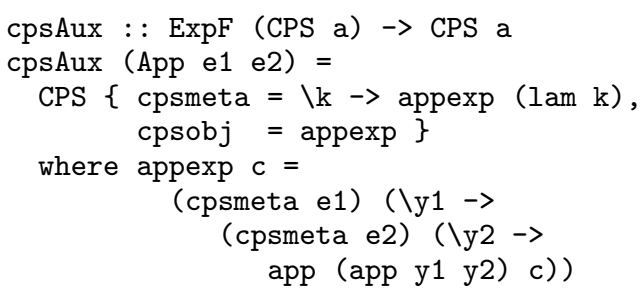

\footnotetext{
${ }^{8}$ In Haskell, the notation apply $\mathrm{x}$ projects the apply component from the record $\mathrm{x}$.
} 


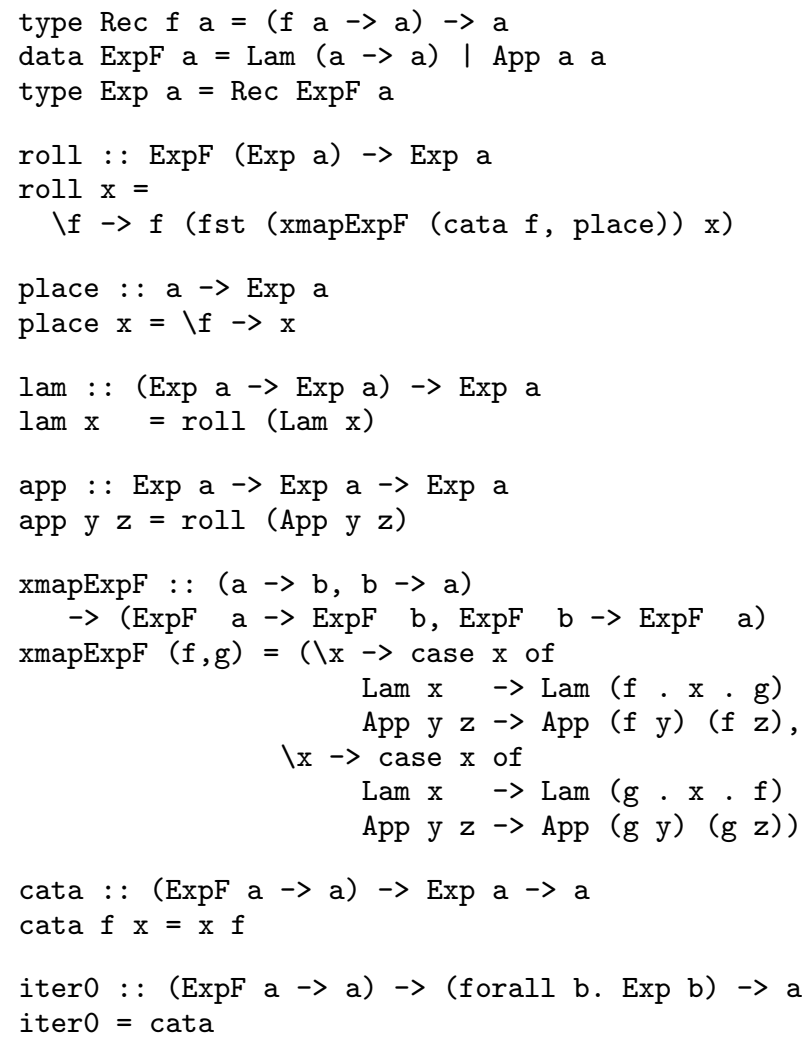

Figure 4. Catamorphism in the $\mathrm{F}_{\omega}$ fragment of Haskell

For functions, we use value, but we must transform the function to bind both the original and continuation arguments and transform the body of the function to use this object continuation. The outer lam binds the original argument. We use value for this argument in $f$ and cpsobj yields a body expecting an object continuation that the inner lam converts to an expression.

$\operatorname{cpsAux}(\operatorname{Lam} f)=$

value (lam (lam. cpsobj.f. value))

Finally, we start cps with iter0 by abstracting an arbitrary dynamic context a and transforming the argument with respect to that context.

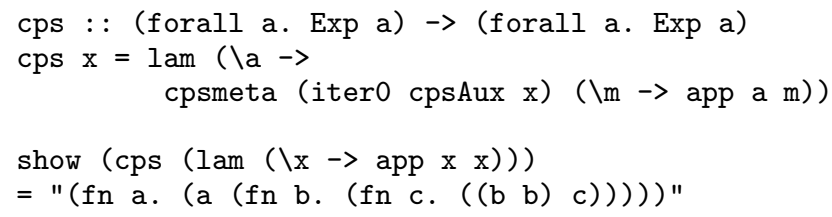

Above, $\mathrm{a}$ is the initial continuation, $\mathrm{b}$ is the $\operatorname{argument} \mathrm{x}$, and $\mathrm{c}$ is the continuation for the function.

\section{Encoding iteration in $\mathrm{F}_{\omega}$}

In the previous section, we implemented iter as a recursive function and used a recursive type, Rec, to define Exp. To prevent case analysis, we hid this definition of Rec behind a module boundary. However, this module abstraction and is not the only way to prevent case analysis. Furthermore, term and type recursion is not neces- sary to implement this datatype. We may define iter and Rec in the fragment of Haskell that corresponds to $F_{\omega}[10]$ so that iteration is the only elimination form for Rec. This implementation appears in Figure 4.

The encoding is similar to the encoding of covariant datatypes in the polymorphic $\lambda$-calculus [3] (or to the encoding of Church numerals). We encode an expression of type Exp a as its elimination form. For example, something of type Exp a should take an elimination function of type $(\operatorname{ExpF} a \rightarrow$ a) and return an $a$. To implement cata we apply the expression to the elimination function.

To create an expression, roll must encode this elimination. Therefore, roll returns a function that applies its argument $f$ (the elimination function) to the result of iterating over x. Again, to use xmap we need a right inverse for cata $f$. The term place in Figure 4 is an expression that when analyzed returns its argument. We can show that place is a right inverse by expanding the above definitions:

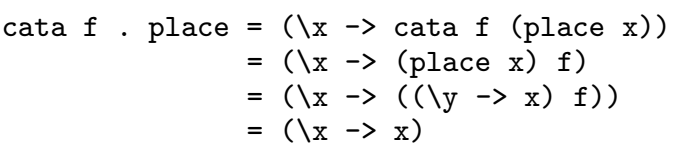

\subsection{Reasoning about iteration}

There are powerful tools for reasoning about programs written in the polymorphic $\lambda$-calculus. For example, we know that all programs that are written in $\mathrm{F}_{\omega}$ will terminate. Therefore, we can argue that the examples of the previous section are total on all inputs that may be expressed in the polymorphic $\lambda$-calculus, such as app $(\operatorname{lam}(\backslash \mathrm{x} \rightarrow \operatorname{app} \mathrm{x} x))(\operatorname{lam}(\backslash \mathrm{x} \rightarrow$ app $\mathrm{x} x))$. Unfortunately, we cannot argue that these examples are total for arbitrary Haskell terms. For example, calling any of these routines on ( $\operatorname{lam}$ (let $f x=f x$ in $f$ )) will certainly diverge. Furthermore, even if the arguments to iteration are written in $\mathrm{F}_{\omega}$, if the operation itself uses type or term recursion, then it could still diverge. For example, using the recursive datatype Value from Section 2, we can implement the untyped $\lambda$-calculus evaluator with iter0.

Parametricity is another way to reason about programs written in $\mathrm{F}_{\omega}$. As awkward as they may be, one of the advantages to programming with catamorphisms instead of general recursion is that we may reason about our programs using algebraic laws that follow from parametricity. While the following laws only hold for $F_{\omega}$, we may be able to prove some form of them for Haskell using techniques developed by Johann [12].

Using parametricity, we can derive a free theorem [28] about expressions of type (forall a. (b a $\rightarrow$ a) $\rightarrow$ a). If $x$ has this type, then

$f \cdot f^{\prime}=i d$ and $f \cdot g=h$. fst $\left(x \operatorname{xap}|b|\left(f, f^{\prime}\right)\right) \Rightarrow$ $f(x g)=x h$

The equivalence in this theorem is equivalence in some parametric model of $F_{\omega}$, such as the term model with $\beta \eta$-equivalence. Using the free theorem, we can prove a number of properties about iteration. First, we can show that iterating roll is an identity function, that iter0 roll = id. Using this result we can show the uniqueness property for iter, which describes when a function is equal to an application of iter. It resembles an "induction principle" for iter0. 
$f \cdot f^{\prime}=i d$ and $f \cdot \operatorname{roll}=h$. fst $\left(\operatorname{xmap}|b|\left(f, f^{\prime}\right)\right)$ $\Leftrightarrow$ f $=$ iter0 $\mathrm{h}$

The <= direction follows directly from the implementation of iter 0 and roll. The $=>$ direction follows from the free theorem.

Finally, the fusion law can be used to combine the composition of a function $f$ and an iteration into one iteration. This law follows directly from the free theorem.

$f \cdot f^{\prime}=i d$ and $f \cdot g=h \cdot f \operatorname{st}\left(x \operatorname{xmap}|b|\left(f, f^{\prime}\right)\right) \Rightarrow$ $f$. iter0 $g=i t e r 0 h$

However, there is an important property about this encoding of the $\lambda$-calculus that we have not proven. Adequacy states that if a $\mathrm{F}_{\omega}$ term is of type forall a. Exp a and is in canonical form, then it should be the encoding of the canonical form of some $\lambda$-calculus expression. In other words, there is no extra "junk" in the type forall a. Exp a, such as badcase. As a first step towards proving this result, we next show how this $\mathrm{F}_{\omega}$ library can encode a language with iteration over HOAS that itself adequately embeds the $\lambda$-calculus.

\section{Enforcing parametricity with modal types}

In the next section, we formally describe the connection between the interface we have provided for iteration over higher-order abstract syntax and the modal calculus of Schürmann, Despeyroux and Pfenning (SDP) [24]. We do so by using this library to give a sound and complete embedding of the SDP calculus into $\mathrm{F}_{\omega}$. First, we provide a brief overview of the static and dynamic semantics of this calculus. The syntax of the SDP calculus is shown in Figure 5.

The SDP calculus enforces the parametricity of function spaces with modal types. Modal necessity in logic is used to indicate those propositions that are true in all worlds. Consequently, these propositions can make use of only those assumptions that are also true in all worlds. In Pfenning and Davies' [20] interpretation of modal necessity, necessarily true propositions correspond to those formulae that can be shown to be valid. Validity is defined as derivable with respect to only assumptions that themselves are valid assumptions. As such, the typing judgments have two environments (also called contexts), one for valid assumptions, $\Omega$, and one for "local" assumptions, $\Upsilon$. The terms corresponding to the introduction and elimination forms for modal necessity are box and let box. We give them the following typing rules:

$$
\begin{gathered}
\frac{\Omega ; \varnothing \vdash M: A}{\Omega ; \Upsilon \vdash \operatorname{box} M: \square A} \\
\frac{\Omega ; \Upsilon \vdash M_{1}: \square A_{1} \quad \Omega \uplus\left\{x: A_{1}\right\} ; \Upsilon \vdash M_{2}: A_{2}}{\Omega ; \Upsilon \vdash \text { let box } x: A_{1}=M_{1} \operatorname{in} M_{2}: A_{2}}
\end{gathered}
$$

A boxed term, $M$, has type $\square A$ only if it has type $A$ with respect to the valid assumptions in $\Omega$, and no assumptions in local environment. The let box elimination construct allows for the introduction of valid assumptions into $\Omega$, binding the contents of the boxed term $M_{1}$ in the body $M_{2}$. This binding is allowed because the contents of boxed terms are well-typed themselves with only valid assumptions. Another way to think about modal necessity is that terms with boxed type are "closed" and do not contain any free variables, except those that are bound to closed terms themselves.

Operationally, boxed terms behave like suspensions, while let box substitutes the contents of a boxed term for the bound variable. Because the operational semantics is defined simultaneously with con-

$$
\begin{array}{lrl}
\text { (Pure Types) } & B::=b|1| B_{1} \rightarrow B_{2} \mid B_{1} \times B_{2} \\
\text { (Types) } & A::=B\left|A_{1} \rightarrow A_{2}\right| A_{1} \times A_{2} \mid \square A \\
\text { (Terms) } & M::=x|c|\langle\rangle|\lambda x: A . M| M_{1} M_{2} \mid \\
& & \text { box } M \mid \text { let box } x: A=M_{1} \text { in } M_{2} \mid \\
& & \left\langle M_{1}, M_{2}\right\rangle|\mathbf{f s t} M| \text { snd } M \mid \\
& & \text { iter }\left[A_{1}, A_{2}\right][\Theta] M \\
\text { (Term Replacement) } & \Theta::=\varnothing|\Theta \uplus\{x \mapsto M\}| \Theta \uplus\{c \mapsto M\} \\
\text { (Pure Environment) } & \Psi::=\varnothing \mid \Psi \uplus\{x: B\} \\
\text { (Valid Environment) } & \Omega::=\varnothing \mid \Omega \uplus\{x: A\} \\
\text { (Local Environment) } & \Upsilon::=\varnothing \mid \Upsilon \uplus\{x: A\} \\
\text { (Signatures) } & \Sigma::=\varnothing \mid \Sigma \uplus\{c: B \rightarrow b\} &
\end{array}
$$

\section{Figure 5. Syntax of SDP calculus}

version to canonical forms, it is parameterized by the environment $\Psi$ that describes the types of free local variables appearing in the expression.

$$
\frac{\Psi \vdash M_{1} \hookrightarrow \operatorname{box} M_{1}^{\prime}: \square A_{1} \quad \Psi \vdash M_{2}\left\{M_{1}^{\prime} / x\right\} \hookrightarrow V: A_{2}}{\Psi \vdash \operatorname{let} \operatorname{box} x: A_{1}=M_{1} \operatorname{in} M_{2} \hookrightarrow V: A_{2}}
$$

To enforce the separation between the iterative and parametric function spaces, the SDP calculus defines those types, $B$, that do not contain a $\square$ type to be "pure". Objects in the calculus with type $\square B$, boxed pure types, can be examined intensionally using an iteration operator, while objects of arbitrary impure type, $A$, cannot. This forces functions of pure type, $\lambda x: B_{1} . . M: B_{1} \rightarrow B_{2}$, to be parametric. This is because the input, $x$, to such a function does not have a boxed pure type, and there is no way to convert it to one $-x$ will not be free inside of a boxed expression in $M$. Consequently, the functions of pure type may only treat their inputs extensionally, making them parametric.

The language is parameterized by a constant type $b$ and a signature, $\Sigma$, of data constructor constants, $c$, for that base type. Each of the constructors in this signature must be of type $B \rightarrow b$. Because $B$ is a pure type, these constructors may only take parametric functions as arguments.

For example, consider a signature describing the untyped $\lambda$ calculus, $\Sigma=\{$ app $: b \times b \rightarrow b$, lam $:(b \rightarrow b) \rightarrow b\}$, where the constant type $b$ corresponds to Exp. Using this signature, we can write a function to count the number of bound variables in an expression, as we did in Section $2 .^{9}$

$$
\begin{aligned}
& \text { countvar } \triangleq \lambda x: \square b . \\
& \operatorname{iter}[\square b, \text { int }][\{\mathbf{a p p} \mapsto \lambda y: \text { int. } \lambda z: \text { int. } y+z \text {, } \\
& \text { lam } \mapsto \lambda f: \text { int } \rightarrow \text { int. } f 1\}] x
\end{aligned}
$$

The term iter intensionally examines the structure of the argument $x$ and replaces each occurrence of app and lam with $\lambda y:$ int. $\lambda z:$ int. $y+z$ and $\lambda f:$ int $\rightarrow$ int. $f 1$ respectively.

The typing rule for iter is the following:

$$
\frac{\Omega ; \Upsilon \vdash M: \square B \quad \Omega ; \Upsilon \vdash \Theta: A\langle\Sigma\rangle}{\Omega ; \Upsilon \vdash \operatorname{iter}[\square B, A][\Theta] M: A\langle B\rangle}
$$

The argument to iteration, $M$, must have a pure closed type to be analyzable. Analysis proceeds via walking over $M$ and using the

\footnotetext{
${ }^{9}$ For simplicity, our formal presentation of SDP (in Figure 5) does not include integers. However, it is straightforward to extend this calculus to additional base types.
} 
replacement $\Theta$, a finite map from constants to terms, to substitute for the constants in the term $M$. The type $A$ is the type that will replace the base type $b$ in the result of iteration. The notation $A\langle B\rangle$ substitutes $A$ for the constant $b$ in the pure type $B$. Each term in the range of the replacements must also agree with replacing $b$ with $A$. We verify this fact with the judgment $\Omega ; \Upsilon \vdash \Theta: A\langle\Sigma\rangle$, which requires that if $\Theta(c)=M_{c}$ and $\Sigma(c)=B_{c}$, then $M_{c}$ must have type $A\left\langle B_{c}\right\rangle$.

Operationally, iteration in the SDP calculus works in the following fashion.

$$
\begin{gathered}
\Psi \vdash M \hookrightarrow \operatorname{box} M^{\prime}: \square B \\
\varnothing \vdash M^{\prime} \Uparrow V^{\prime}: B \\
\Psi \vdash\langle A, \Psi, \Theta\rangle\left(V^{\prime}\right) \hookrightarrow V: A\langle B\rangle \\
\frac{\Psi \vdash \operatorname{iter}[\square B, A][\Theta] M \hookrightarrow V: A\langle B\rangle}{\square]}
\end{gathered}
$$

First, the argument to iteration $M$ is evaluated, $\Psi \vdash M \hookrightarrow \operatorname{box} M^{\prime}$ : $\square B$, producing a boxed object $M^{\prime} . M^{\prime}$ is then evaluated to $\eta$-long canonical form via $\varnothing \vdash M^{\prime} \Uparrow V^{\prime}: B$. Next we perform elimination of that canonical form, $\langle A, \Psi, \Theta\rangle\left(V^{\prime}\right)$, walking over $V^{\prime}$ and using $\Theta$ to replace the occurrences of constants. Finally, we evaluate that result, $\Psi \vdash\langle A, \Psi, \Theta\rangle\left(V^{\prime}\right) \hookrightarrow V: A\langle B\rangle$.

In order to simplify the presentation of the encoding, we have made a few changes to the SDP calculus. First, while the language presented in this paper has only one pure base type $b$, the SDP calculus allows the signature $\Sigma$ to contain arbitrarily many base types. However, the extension of the encoding to several base types is straightforward. Also, in order to make the constants of the pure language more closely resemble datatype constructors, we have forced them all to be of the form $B \rightarrow b$ instead of any arbitrary pure type $B$. To facilitate this restriction, we add unit and pairing to the pure fragment of the calculus so that constructors may take any number of arguments.

\section{Encoding SDP in $\mathrm{F}_{\omega}$}

The terms that we defined in Section 3, roll and iter, correspond very closely to the constructors and iteration primitive of the SDP calculus. In this section, we strengthen this observation by showing how to encode all programs written in the SDP calculus into $F_{\omega}$ using a variation of these terms.

\section{There are two key ideas behind our encoding:}

- We use type abstraction to ensure that the encoding of boxed objects obeys the closure property of the source language, and prevents variables from the local environment from appearing inside these terms. To do so, we parameterize our encoding by a type that represents the current world and maintain the invariant that all variables in the local environment mention the current world in their types. Because a term enclosed within a box must be well-typed in any world, when we encode a boxed term we use a fresh type variable to create an arbitrary world. We then encode the enclosed term with that new world and wrap the result with a type abstraction. As a consequence, the encoding of a data-structure within a box cannot contain free local variables because their types would mention that fresh type variable outside of the scope of the type abstraction.

- We encode constants in the source language as their elimination form with roll. Furthermore, we restrict the result of elimination to be of the type that is the world in which the term was encoded. However, the encoding of boxed expres- sions quantifies over that world, allowing the resulting computations to be of arbitrary type.

The encoding of the SDP calculus can be broken into four primary pieces: the encodings for signatures, types, terms, and replacements. To simplify our presentation, we extend the target language with unit, void, products, and variants. The syntax of these terms appears in Figure 6. This extension does not weaken our results as there are well known encodings of these types into $F_{\omega}$. In the remainder of this section, we present the details of the encoding and describe the most interesting cases. The full specification of this encoding appears in Appendix B.

Signatures. The encoding of signatures in the SDP calculus, notated $\tau\langle\Sigma\rangle$, corresponds to generating the type constructor whose fixed point defines the recursive datatype. (For example, ExpF in Section 2.) The argument of the encoding, a specified world $\tau$, corresponds to the argument of the type constructor.

For this encoding, we assume the aid of an injective function $\mathcal{L}$ that maps data constructors in the source language to distinct labels in the target language. We also need an operation called parameterization, notated $\tau\langle B\rangle$ and defined in Appendix B.1. This operation parameterizes pure types in the source calculus with respect to a given world in the target language, and produces a type in the target language. Essentially, $\tau\langle B\rangle$ "substitutes" the type $\tau$ for the base type, $b$, in $B$.

We encode a signature as a variant. Each field corresponds to a constant $c_{i}$ in the signature, with a label according to $\mathcal{L}$, and a type that is the result of parameterizing the argument type of $c_{i}$ with the provided type.

$$
\frac{\forall c_{i} \in \operatorname{dom}(\Sigma) \quad \Sigma\left(c_{i}\right)=B_{i} \rightarrow b}{\tau\langle\Sigma\rangle \triangleq\left\langle\mathcal{L}\left(c_{1}\right): \tau\left\langle B_{1}\right\rangle, \ldots, \mathcal{L}\left(c_{n}\right): \tau\left\langle B_{n}\right\rangle\right\rangle}
$$

We often use parameterization and the signature translation to build type constructors in the target language, so we define the following two abbreviations:

$$
B^{*} \triangleq \lambda \alpha: \star . \alpha\langle B\rangle \quad \Sigma^{*} \triangleq \lambda \alpha: \star . \alpha\langle\Sigma\rangle
$$

Types. As with the encoding of signatures, the encoding of types is parameterized by the worlds in which they occur. We write the judgment for encoding a type $A$ in the source calculus in world $\tau$ as $\Delta \vdash A \triangleright_{\tau} \tau^{\prime}$. The environment $\Delta$ tracks type variables allocated during the translation and allows us to chose variables that are not in scope. The two interesting cases for encoding types from the source calculus are those for the base type and for boxed types. The case for $b$ corresponds to Rec $\operatorname{ExpF}$ a from Section 3. Therefore, we define the abbreviation $\operatorname{Rec} \Sigma^{*} \alpha \triangleq\left(\Sigma^{*} \alpha \rightarrow \alpha\right) \rightarrow \alpha$, intuitively a fixed point of $\Sigma^{*}$, to the same idea of encoding a datatype as its elimination form.

$$
\overline{\Delta \vdash b \triangleright_{\tau} \operatorname{Rec} \Sigma^{*} \tau}
$$

The rule for boxed types uses type abstraction to ensure the result is parametric with respect to its world. Naïvely, we might expect to use a fresh type variable as the new world and then encode the contents of the boxed type with that type variable. This encoding ensures that the type is parametric with respect to its world and then quantifies over the result.

$$
\frac{\alpha \notin \Delta \quad \Delta \uplus\{\alpha: \star\} \vdash A \triangleright_{\alpha} \tau^{\prime}}{\Delta \vdash \square A \triangleright_{\tau} \forall \alpha: \star . \tau^{\prime}} \text { WRONG! }
$$


(Kinds)

(Types)

(Terms)

(Type Variable Environment)

(Term Environment)

$$
\begin{aligned}
& \kappa::=\star \mid \kappa_{1} \rightarrow \kappa_{2} \\
& \tau::=1|0| \alpha\left|\tau_{1} \rightarrow \tau_{2}\right| \forall \alpha: \kappa . \tau\left|\tau_{1} \times \tau_{2}\right|\left\langle l_{1}: \tau_{1}, \ldots, l_{n}: \tau_{n}\right\rangle|\lambda \alpha: \kappa . \tau| \alpha \mid \tau_{1} \tau_{2} \\
& e::=x|\langle\rangle| \lambda x: \tau . e\left|e_{1} e_{2}\right| \Lambda \alpha: \kappa . e|e[\tau]|\left\langle e_{1}, e_{2}\right\rangle \mid \text { fst } e \mid \text { snd } e \mid \mathbf{i n j}_{l} e \text { of } \tau \mid \\
& \text { case } e \text { of inj } j_{l_{1}} x_{1} \text { in } e_{1} \ldots \text { inj }_{l_{n}} x_{n} \text { in } e_{n} \\
& \Delta::=\varnothing \mid \Delta \uplus\{\alpha: \kappa\} \\
& \Gamma::=\varnothing \mid \Gamma \uplus\{x: \tau\}
\end{aligned}
$$

Figure 6. Syntax of $F_{\omega}$ with unit, void, products, and variants
However, with this encoding we violate the invariant that the types of all free local variables mention the current world, because the encoding does not involve $\tau$. Instead, we use the fresh type variable to create a new world from the current world and consider $\alpha$ as a "world transformer". During the translation, a term will be encoded with a stack of world transformers, somewhat akin to stack of environments in the implicit formulation of modal types [7].

$$
\frac{\alpha \notin \Delta \quad \Delta \uplus\{\alpha: \star \rightarrow \star\} \vdash A \triangleright_{\alpha \tau} \tau^{\prime}}{\Delta \vdash \square A \triangleright_{\tau} \forall \alpha: \star \rightarrow \star . \tau^{\prime}}
$$

The naïve translation of the unit type also forgets the current world. For this reason, we add a non-standard unit to $\mathrm{F}_{\omega}$ that is parameterized by the current world. In other words, the unit type 1 is of kind $\star \rightarrow \star$ and the unit term \langle\rangle has type $\forall \alpha: \star .1(\alpha)$. Our type translation instantiates this type with the current world.

$$
\overline{\Delta \vdash 1 \triangleright_{\tau} 1[\tau]}
$$

The remaining types in the SDP language are encoded recursively in a straightforward manner. The complete rules can be found in Appendix B.3.

Terms and replacements. We encode the source term, $M$, with the judgment $\Delta ; \Xi \vdash M \triangleright_{\tau} e$. In addition to the current world, $\tau$, and the set of allocated type variables, $\Delta$, the encoding of terms is also parameterized by a set of term variables, $\Xi$. This set of variables allows the encoding to distinguish between variables that were bound with $\lambda$ and those bound with let box. We will elaborate on why this set is necessary shortly.

Our encoding of boxed terms follows immediately from the encoding of boxed types. Here we encode the argument term with respect to a fresh world transformer applied to the present world and then wrap the result with a type abstraction.

$$
\frac{\alpha \notin \Delta \quad \Delta \uplus\{\alpha: \star \rightarrow \star\} ; \Xi \vdash M \triangleright_{\alpha \tau} e}{\Delta ; \Xi \vdash \operatorname{box} M \triangleright_{\tau} \Lambda \alpha: \star \rightarrow \star . e}
$$

We encode let box by converting it to an abstraction and application in the target language. However, one might note the discrepancy between the type of the variable we bind in the abstraction and the type we might naïvely expect.

$$
\begin{gathered}
\Delta \vdash \square A_{1} \triangleright_{\tau} \tau_{1} \\
\frac{\Delta ; \Xi \vdash M_{1} \triangleright_{\tau} e_{1} \quad \Delta ; \Xi \uplus\{x\} \vdash M_{2} \triangleright_{\tau} e_{2}}{\Delta ; \Xi \vdash \text { let box } x: A_{1}=M_{1} \operatorname{in} M_{2} \triangleright_{\tau}\left(\lambda x: \tau_{1} . e_{2}\right) e_{1}}
\end{gathered}
$$

The type of $x$ is $A_{1}$ and so one might assume that the type of $x$ in the target should be the encoding of $A_{1}$ in the world $\tau$. However, let box allows us to bind variables that are accessible in any world and using $A_{1}$ encoded against $\tau$ would allow the result to be used

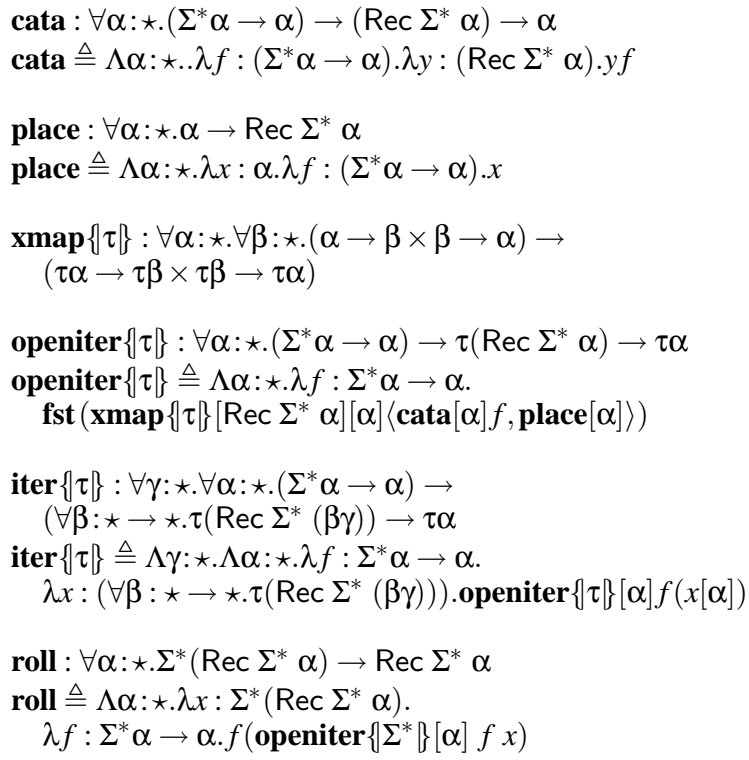

Figure 7. Library routines

only in the present world. Because the encoding of $M_{1}$ will evaluate to a type abstraction, a term parametric in its world, we do not immediately unpack it by instantiating it with the current world. Instead we pass it as $x$ and then, when $x$ appears we instantiate it with the current world. Consequently, we use $\Xi$ to keep track of variables bound by let box. When encoding variables, we check whether $x$ occurs in $\Xi$ and perform instantiations as necessary.

$$
\frac{x \notin \Xi}{\Delta ; \Xi \vdash x \triangleright_{\tau} x} \quad \frac{x \in \Xi}{\Delta ; \Xi \vdash x \triangleright_{\tau} x[\lambda \alpha: \star . \tau]}
$$

If the variable is in $\Xi$, then it is applied to a world transformer that ignores its argument, and returns the present world. This essentially replaces the bottom of the world transformer stack captured by the type abstraction substituted for $x$ with the world $\tau$. Doing so ensures that if we substitute the encoding of a boxed term into the encoding of another boxed term, the type correctness of the embedding is maintained by correctly propagating the enclosing world.

Figure 7 shows the types and definitions of the library routines used by the encoding. The only difference between it and Figure 4 is that iter abstracts the current world and requires that its argument be valid in any transformation of the current world. Again, we make use of the polytypic function xmap to lift cata to arbitrary type constructors . Because xmap is defined by the structure of a type constructor $\tau$, we cannot directly define it as a term in $F_{\omega}$. Instead, we will think of $\operatorname{xmap}\{\tau\}$ as macro that expands to the 
mapping function for the type constructor $\tau$. (We use the notation $\{\cdot\}$ to distinguish between polytypic instantiation and parametric type instantiation.) This expansion is done according to the definition in Appendix A. We do not cover the implementation here, see Hinze [11] for details.

Encoding constants in the source calculus makes straightforward use of the library routine roll. We simply translate the constant into an abstraction that accepts a term that is the encoding of the argument of the constant, and then uses roll to transform the injection into the encoding of the base type, $\operatorname{Rec} \Sigma^{*} \tau$.

$$
\frac{\Sigma(c)=B \rightarrow b \quad \Delta \vdash B \triangleright_{\tau} \tau_{B}}{\Delta ; \Xi \vdash c \triangleright_{\tau} \lambda x: \tau_{B} \cdot \operatorname{roll}[\tau]\left(\operatorname{inj}_{\mathcal{L}(c)} x \text { of } \Sigma^{*}\left(\operatorname{Rec} \Sigma^{*} \tau\right)\right)}
$$

The encoding of iteration is similarly straightforward. We instantiate our polytypic function iter with a type constructor created from parameterizing $B$, and then apply it to the current world and the encodings of the intended result type $A$, the replacement term $\Theta$ and argument term $M$.

$$
\frac{\Delta \vdash A \triangleright_{\tau} \tau_{A} \quad \Delta ; \Xi \vdash \Theta \triangleright_{\tau}^{\tau_{A}} e_{\Theta} \quad \Delta ; \Xi \vdash M \triangleright_{\tau} e_{M}}{\Delta ; \Xi \vdash \operatorname{iter}[\square B, A][\Theta] M \triangleright_{\tau} \operatorname{iter}\left\{B^{*}\right\}[\tau]\left[\tau_{A}\right] e_{\Theta} e_{M}}
$$

The encoding of replacements $\Theta$ is uncomplicated and analogous to the encoding of signatures. We construct an abstraction that consumes an instance of an encoded signature, dispatching the variant using a case expression. In each branch, the encoding of the corresponding replacement is applied to the argument of the injection.

$$
\begin{array}{r}
\forall c_{i} \in \operatorname{dom}(\Theta) \quad \Delta ; \Xi \vdash \Theta\left(C_{i}\right) \triangleright_{\tau} e_{i} \\
\Delta ; \Xi \vdash \Theta \triangleright \tau_{\tau}^{\tau_{A}} \lambda x: \Sigma^{*} \tau_{A} \cdot \operatorname{case} x \mathbf{o f i n j}_{\mathcal{L}\left(c_{1}\right)} y_{1} \text { in }\left(e_{1} y_{1}\right) \\
\ldots \\
\mathbf{i n j}_{\mathcal{L}\left(c_{n}\right)} y_{n} \text { in }\left(e_{n} y_{n}\right)
\end{array}
$$

The encodings for the other terms in the source language are straightforward and appear in Appendix B.4. Now that we have defined all of our encoding for any closed term $M$ in the SDP calculus, we put everything together to construct a term $e$ in our target calculus using the initial judgment $\varnothing ; \varnothing \vdash M \triangleright_{0} e$. We use the void type as the initial world to enforce the parametricity of unboxed constants.

\subsection{Properties of the encoding}

We have proven a number of desirable properties concerning this encoding. However, before we can state these properties, we must first define the relationship between the environments in the source and target calculi. These relations hold when all types from the local environment are encoded with the current world, and all types from the valid environment are first boxed then encoded with any world.

DEFINITION 5.1 (ENCODING TYPING ENVIRONMENTS). We write $\Delta \vdash \Upsilon \triangleright_{\tau} \Gamma_{1}$ and $\Delta \vdash \Omega \triangleright \Gamma_{2}$ to mean that

$$
\begin{array}{cc}
\forall x: A \in \Upsilon, x: \tau_{A} \in \Gamma_{1} & \text { when } \Delta \vdash A \triangleright_{\tau} \tau_{A} \\
\forall x: A \in \Omega, x: \tau_{A} \in \Gamma_{2} & \text { when there exists } \Delta \vdash \tau^{\prime} \text { such that } \\
\Delta \vdash \square A \triangleright_{\tau^{\prime}} \tau_{A}
\end{array}
$$

The relation for valid environments above is not parameterized by the current world. A single valid environment may be encoded as many different target environments, depending on what worlds are chosen for each type in the environment. However, in some sense the encodings are equivalent. If the translation of $M$ type checks with one encoding of $\Omega$, it will type check with any other encoding of $\Omega$.

The encoding is type preserving. If we encode a well-typed term $M$, the resulting term will be well-typed under the appropriately translated environment. Furthermore, the converse is also true. If the encoding of a term $M$ is well-typed in the target language, then $M$ must have been well-typed in the source. This means that the target language preserves the abstractions of the source language.

THEOREM 5.2 (STATIC CORRECTNESS). Assume $\Delta \vdash \tau$ and $\Delta \vdash \Upsilon \triangleright_{\tau} \Gamma_{1}$ and $\Delta \vdash \Omega \triangleright \Gamma_{2}$.

$$
\begin{aligned}
& \text { 1. If } \Delta ; \operatorname{dom}(\Omega) \vdash M \triangleright_{\tau} e \text { then } \\
& \Omega ; \Upsilon \vdash M: A \text { and } \Delta \vdash A \triangleright_{\tau} \tau_{A} \Leftrightarrow \Delta ; \Gamma_{1} \uplus \Gamma_{2} \vdash e: \tau_{A}
\end{aligned}
$$

2. If $\Delta ; \operatorname{dom}(\Omega) \vdash \Theta \triangleright \tau_{\tau}^{\tau_{A}} e_{\theta}$ then

$\Omega ; \Upsilon \vdash \Theta: A\langle\Sigma\rangle$ and $\Delta \vdash A \triangleright_{\tau} \tau_{A} \Leftrightarrow \Delta ; \Gamma_{1} \uplus \Gamma_{2} \vdash e_{\theta}: \tau_{A}\langle\Sigma\rangle \rightarrow \tau_{A}$

Proof. By mutual induction over the translation of terms $\left(\Delta ; \operatorname{dom}(\Omega) \vdash M \nabla_{\tau} e\right)$ and of replacements $\left(\Delta ; \operatorname{dom}(\Omega) \vdash \Theta \triangleright{ }_{\tau}^{\tau_{A}} e_{\theta}\right)$.

Furthermore, source evaluation and canonicalization is the same as $\beta \eta$-equivalence in the target calculus.

THEOREM 5.3 (DyNAMIC CORRECTNESS). If $\varnothing ; \Psi \vdash M: A$ and $\varnothing ; \Psi \vdash M \triangleright_{\tau} e$ and $\varnothing ; \Psi \vdash V \triangleright_{\tau} e^{\prime}$ and $\varnothing \vdash A \triangleright_{\tau} \tau_{A}$ and $\varnothing ; \Xi \vdash \varnothing ; \Psi \triangleright_{\tau} \Gamma$ then

$$
\begin{aligned}
& \text { 1. } \Psi \vdash M \hookrightarrow V: A \Leftrightarrow \varnothing ; \Gamma \vdash e \equiv_{\beta \eta} e^{\prime}: \tau_{A} . \\
& \text { 2. } \Psi \vdash M \Uparrow V: A \Leftrightarrow \varnothing ; \Gamma \vdash e \equiv_{\beta \eta} e^{\prime}: \tau_{A} .
\end{aligned}
$$

PROOF. The forward direction follows by simultaneous induction on the evaluation of $M(\Psi \vdash M \hookrightarrow V: A)$ and the conversion of $M$ to canonical form $(\Psi \vdash M \Uparrow V: A)$. The reverse direction follows from the forward direction and from the fact that evaluation in the SDP calculus is deterministic and total.

\section{Future work}

Although we have shown a very close connection between SDP and its encoding in $F_{\omega}$, we have not shown that this encoding is adequate. We would like to show that if $\tau$ is the image of an SDP type, then all terms of type $\tau$ are equivalent to the encoding of some SDP term. In other words, there is no extra "junk" of type $\tau$. Showing this result would also show that encoding the $\lambda$-calculus with app and lam is adequate, because the SDP calculus can already adequately encode the $\lambda$-calculus.

Alternatively, we could try to show adequacy with respect to the $\lambda$ calculus directly using a different method. It may also be possible to do so for the simpler encoding of modal types, informally presented in the first part of the paper, that uses first-order quantification and discards the current world. Whereas this simpler encoding allows the translation of some terms that are rejected by the SDP calculus to type check (for example, $\lambda x: \square b$. box $x$ ), it may still be adequate for encoding the untyped $\lambda$-calculus.

One important extension of this work is the case operator. Because there are limitations to what may be defined with iter, the SDP calculus also includes a construct for case analysis of closed terms. 
However, we have not yet found an obvious correspondence for case in our encoding.

Another further area of investigation is into the dual operation to iter, the anamorphism over datatypes with embedded functions. An implementation of this operation, called coiter, is below. The coiter term is an anamorphism-it generates a recursive data structure from an initial seed.

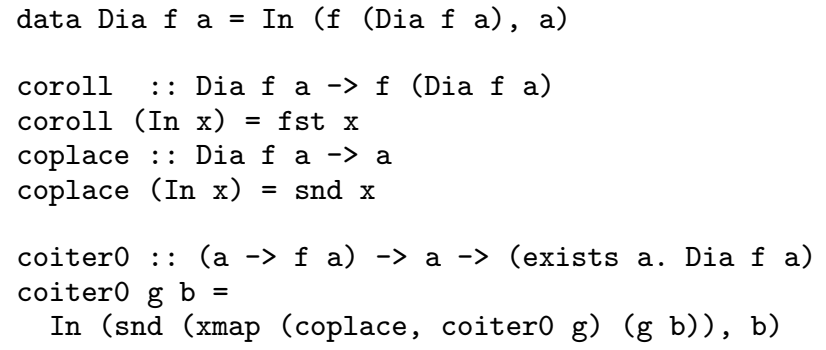

Instead of embedding the recursive type in a sum, we embed it in a product. The two selectors from this product have the dual types to roll and place. In the definition of coiter 0 we use coplace as the inverse where we would have used cata in the definition of ana. A term of type (exists a. Dia b a) corresponds to the possibility type $(\diamond b)$ in a modal calculus. However, while a general anamorphism is an inverse of a catamorphism, coiter is not an inverse to iter. In fact, iter cannot consume what coiter produces, giving doubts to its practical use. (On the other hand, ana itself has seen little practical use for datatypes with embedded functions.) From a logical point of view, this restriction makes sense. Combining anamorphisms and catamorphisms (even for datatypes without embedded functions) leads to general recursion.

\section{Related work}

The technique we present, using polymorphism to enforce parametricity, has appeared under various guises in the literature. For example, Shao et al. [27] use this technique (one level up) to implement type-level intensional analysis of recursive types. They use higher-order abstract syntax to the represent recursive types and remark that the kind of this type constructor requires a parametric function as its argument. However, they do not make a connection with modal type systems, nor do they extend their type-level iteration operator to higher kinds. Xi et al. [31] remark on the correspondence between HOAS terms with the place operator (which they call HOASvar) and closed terms of Mini-ML ${ }_{e}^{\square}$ but do not investigate the relationship or any form of iteration.

While higher-order abstract syntax has an attractive simplicity, the difficulties programming and reasoning about structures encoded with this technique have motivated research into language extensions for working with higher-order abstract syntax or alternative approaches altogether. Dale Miller developed a small language called $\mathrm{ML}_{\lambda}$ [17] that introduces a type constructor for terms formed by abstracting out a parameter. These types can be thought of as function types that can be intensionally analyzed through pattern matching. Pitts and Gabbay built on the theory of FM-sets to design a language called FreshML [23] that allows for the manipulation and abstraction of fresh "names". Nanevski [18] combines fresh names with modal necessity to allow for the construction of more efficient residual terms, while still retaining the ability to evaluate them at runtime. The Delphin Project [25] by Schürmann et al. develops a functional language for manipulating datatypes that are terms in the LF logical framework. Because higher-order abstract syntax is the primary representation technique in LF, Delphin provides operations for matching over higher-order LF terms in regular worlds. The SDP calculus uses modal necessity to restrict matching to closed worlds, so regular worlds provide additional flexibility without the difficulties of matching in an open world. The Hybrid [2] logical framework provides induction over higher-order abstract syntax by evaluation to de Bruijn terms, which provide straightforward induction.

There is a long history of encoding modality in logic, but only recently has the encoding of modal type systems been explored. Acar et al. [1] use modal types in a functional language that provides control over the use of memoization, and implement it as a library in SML. Because SML does not have modal types or first-class polymorphism, they use run-time checks to enforce the correct use of modality. Davies and Pfenning [7] presented, in passing, a simple encoding of the modal $\lambda$-calculus into the simply-typed $\lambda$-calculus that preserves only the dynamic semantics. Washburn expanded upon this encoding, showing that it bisimulates the source calculus [29].

\section{Conclusion}

While other approaches to defining an induction operator over higher-order abstract syntax require type system extensions to ensure the parametricity of embedded function spaces, the approach that we present in this paper requires only type polymorphism. Because of this encoding, we are able to implement iteration operators for datatypes with embedded parametric functions directly in the Haskell language.

However, despite its simplicity, our approach is equivalent to previous work on induction operators for HOAS. We demonstrate this generality by showing how the modal calculus of Schüermann, Despeyroux and Pfenning may be embedded into $\mathrm{F}_{\omega}$ using this technique. In fact, the analogy of representing boxed terms with polymorphic terms makes semantic sense: a proposition with a boxed type is valid in all worlds and polymorphism makes that quantification explicit.

\section{Acknowledgements}

We thank Margaret DeLap, Eijiro Sumi, Stephen Tse, Stephan Zdancewic and the anonymous ICFP reviewers for providing us with feedback concerning this paper.

\section{References}

[1] U. Acar, G. Blelloch, and R. Harper. Selective memoization. In Thirtieth ACMSIGPLAN-SIGACT Symposium on Principles of Programming Languages, New Orleans, LA, Jan. 2002.

[2] S. Ambler, R. L. Crole, and A. Momigliano. Combining higer order abstract syntax with tactical theorem proving and (co)induction. In Theorem Proving in Higher Order Logics, 15th International Conference, TPHOLs 2002, Hampton, VA, USA, August 20-23, 2002, Proceedings, volume 2410 of Lecture Notes in Computer Science. Springer, 2002.

[3] C. Böhm and A. Berarducci. Automatic synthesis of typed $\Lambda$-programs on term algebras. Theoretical Computer Science, 39:135-154, 1985. 
[4] A. Church. A formulation of the simple theory of types. Journal of Symbolic Logic, 5:56-68, 1940.

[5] D. Clarke, R. Hinze, J. Jeuring, A. Löh, and J. de Wit. The Generic Haskell user's guide. Technical Report UU-CS-200126, Utrecht University, 2001.

[6] O. Danvy and A. Filinski. Representing control: a study of the CPS transformation. Mathematical Structures in Computer Science, 2(4):361-391, Dec. 1992.

[7] R. Davies and F. Pfenning. A modal analysis of staged computation. Journal of the ACM, 48(3):555-604, May 2001.

[8] J. Despeyroux and P. Leleu. Recursion over objects of functional type. Mathematical Structures in Computer Science, 11:555-572, 2001.

[9] L. Fegaras and T. Sheard. Revisiting catamorphisms over datatypes with embedded functions (or, programs from outer space). In Twenty-Third ACMSIGPLAN-SIGACT Symposium on Principles of Programming Languages, pages 284-294, St. Petersburg Beach, FL,USA, 1996.

[10] J.-Y. Girard. Une extension de l'interprétation de Gödel à l'analyse, et son application à l'élimination de coupures dans l'analyse et la théorie des types. In J. E. Fenstad, editor, Proceedings of the Second Scandinavian Logic Symposium, pages 63-92. North-Holland Publishing Co., 1971.

[11] R. Hinze. Polytypic values possess polykinded types. Science of Computer Programming, 43(2-3):129-159, 2002. MPC Special Issue.

[12] P. Johann. A generalization of short-cut fution and its correctness proof. Higher-Order and Symbolic Computation, 15:273-300, 2002.

[13] M. P. Jones. A system of constructor classes: overloading and implicit higher-order polymorphism. Journal of Functional Programming, 5(1), Jan. 1995.

[14] M. P. Jones. Type classes with functional dependencies. In Proceedings of the 9th European Symposium on Programming, ESOP 2000, Berlin, Germany, number 1782 in LNCS. Springer-Verlag, 2000.

[15] E. Meijer, M. M. Fokkinga, and R. Paterson. Functional programming with bananas, lenses, envelopes and barbed wire. In FPCA91: Conference on Functional Programming Languages and Computer Architecture, pages 124-144, 1991.

[16] E. Meijer and G. Hutton. Bananas in space: Extending fold and unfold to exponential types. In FPCA95: Conference on Functional Programming Languages and Computer Architecture, pages 324-333, La Jolla, CA, June 1995.

[17] D. Miller. An extension to ML to handle bound variables in data structures: Preliminary report. In Proceedings of the Logical Frameworks BRA Workshop, May 1990.

[18] A. Nanevski. Meta-programming with names and necessity. In Proceedings of the seventh ACM SIGPLAN international conference on Functional programming, pages 206217. ACM Press, 2002

[19] S. Peyton Jones, editor. Haskell 98 Language and Libraries: The Revised Report. Cambridge University Press, 2003.

[20] F. Pfenning and R. Davies. A judgmental reconstruction of modal logic. Mathematical Structures in Computer Science, 11(4):511-540, Aug. 2001.

[21] F. Pfenning and C. Elliott. Higher-order abstract syntax. In
1988 ACM SIGPLAN Conference on Programming Language Design and Implementation, pages 199-208, Atlanta, GA, USA, June 1988.

[22] F. Pfenning and C. Schürmann. System description: Twelf-a meta-logical framework for deductive systems. In $\mathrm{H}$. Ganzinger, editor, Proceedings of the 16th International Conference on Automated Deduction (CADE-16), pages 202 206, Trento, Italy, July 1999.

[23] A. M. Pitts and M. Gabbay. A metalanguage for programming with bound names modulo renaming. In Mathematics of Program Construction, pages 230-255, 2000.

[24] C. Schürmann, J. Despeyroux, and F. Pfenning. Primitive recursion for higher-order abstract syntax. Theoretical Computer Science, 266(1-2):1-58, Sept. 2001.

[25] C. Schürmann, R. Fontana, and Y. Liao. Delphin: Functional programming with deductive systems. Available at http:// cs-www.cs.yale.edu/homes/carsten/, 2002.

[26] E. Sumii and N. Kobayashi. A hybrid approach to online and offline partial evaluation. Higher-Order and Symbolic Computation, 14(2/3):101-142, 2001.

[27] V. Trifonov, B. Saha, and Z. Shao. Fully reflexive intensional type analysis. In Fifth ACM SIGPLAN International Conference on Functional Programming, pages 82-93, Montreal, Sept. 2000.

[28] P. Wadler. Theorems for free! In FPCA89: Conference on Functional Programming Languages and Computer Architecture, London, Sept. 1989.

[29] G. Washburn. Modal typing for specifying run-time code generation. Available from http://www.cis.upenn.edu/ geoffw/research/, 2001.

[30] S. Weirich. Higher-order intensional type analysis in type-erasure semantics. Available from http://www.cis. upenn.edu/ ${ }^{\text {sweirich/, } 2003 .}$

[31] H. Xi, C. Chen, and G. Chen. Guarded recursive datatype constructors. In Thirtieth ACMSIGPLAN-SIGACT Symposium on Principles of Programming Languages, pages 224-235, New Orleans, LA, USA, Jan. 2003.

\section{A Generic Haskell implementation of xmap}

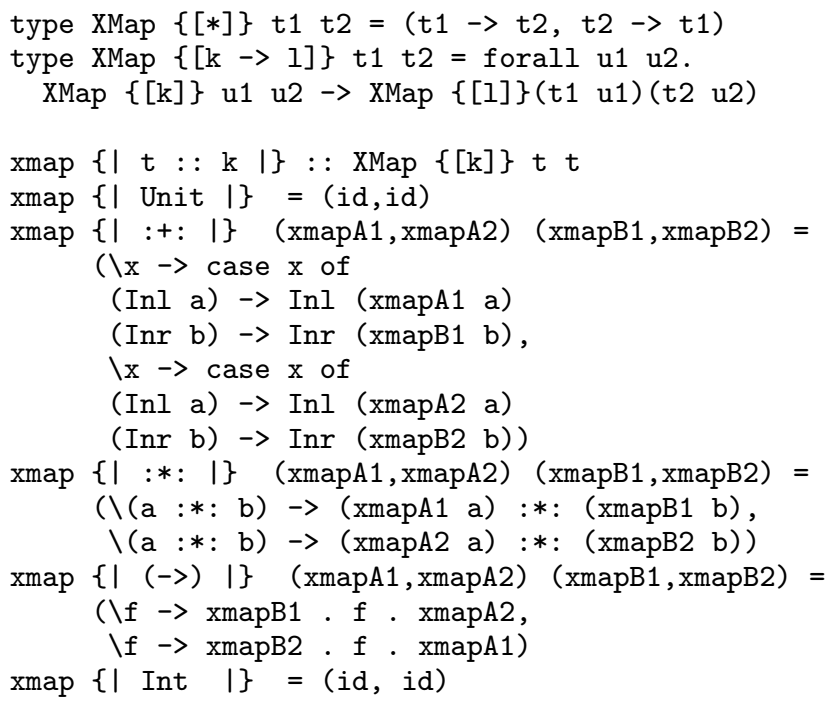


$\operatorname{xmap}\{\mid$ Bool $\mid\}=(i d, i d)$

$\operatorname{xmap}\{\mid$ IO $\mid\}(x m a p A 1, \operatorname{xmapA2})=$

(fmap xmapA1, fmap xmapA2)

$\operatorname{xmap}\{|[]|\} \quad(x m a p A 1, x m a p A 2)=$

(map xmapA1, map xmapA2)

\section{B Full encoding of SDP}

\section{B.1 Parameterization}

$$
\begin{aligned}
& \overline{\tau\langle b\rangle \triangleq \tau} \quad \overline{\tau\langle 1\rangle \triangleq 1} \quad \frac{\tau\left\langle B_{1}\right\rangle \triangleq \tau_{1} \quad \tau\left\langle B_{2}\right\rangle \triangleq \tau_{2}}{\tau\left\langle B_{1} \rightarrow B_{2}\right\rangle \triangleq \tau_{1} \rightarrow \tau_{2}} \\
& \frac{\tau\left\langle B_{1}\right\rangle \triangleq \tau_{1} \quad \tau\left\langle B_{2}\right\rangle \triangleq \tau_{2}}{\tau\left\langle B_{1} \times B_{2}\right\rangle \triangleq \tau_{1} \times \tau_{2}}
\end{aligned}
$$

\section{B.2 Signatures}

$$
\frac{\forall c_{i} \in \operatorname{dom}(\Sigma) \quad \Sigma\left(c_{i}\right)=B_{i} \rightarrow b}{\tau\langle\Sigma\rangle \triangleq\left\langle\mathcal{L}\left(c_{1}\right): \tau\left\langle B_{1}\right\rangle, \ldots, \mathcal{L}\left(c_{n}\right): \tau\left\langle B_{n}\right\rangle\right\rangle}
$$

\section{B.3 Types}

$$
\begin{gathered}
\frac{\Delta \vdash \tau}{\Delta \vdash b \triangleright_{\tau} \operatorname{Rec} \Sigma^{*} \tau} \quad \frac{\alpha \notin \Delta \quad \Delta \uplus\{\alpha: \star \rightarrow \star\} \vdash A \triangleright_{\alpha \tau} \tau^{\prime}}{\Delta \vdash \square A \triangleright_{\tau} \forall \alpha: \star \rightarrow \star . \tau^{\prime}} \\
\frac{\Delta \vdash A_{1} \triangleright_{\tau} \tau_{1} \quad \Delta \vdash A_{2} \triangleright_{\tau} \tau_{2}}{\Delta \vdash 1 \triangleright_{\tau} 1(\tau)} \quad \frac{\frac{\Delta \vdash A_{1} \rightarrow A_{2} \triangleright_{\tau} \tau_{1} \rightarrow \tau_{2}}{\Delta \vdash A_{1} \triangleright_{\tau} \tau_{1} \quad \Delta \vdash A_{2} \triangleright_{\tau} \tau_{2}}}{\Delta \vdash A_{1} \times A_{2} \triangleright_{\tau} \tau_{1} \times \tau_{2}}
\end{gathered}
$$

\section{B.4 Terms}

$$
\begin{aligned}
& \frac{x \notin \Xi}{\Delta ; \Xi \vdash x \triangleright_{\tau} x} \quad \frac{x \in \Xi}{\Delta ; \Xi \vdash x \triangleright_{\tau} x[\lambda \alpha: \star . \tau]} \\
& \frac{\alpha \notin \Delta \quad \Delta \uplus\{\alpha: \star \rightarrow \star\} ; \Xi \vdash M \triangleright_{\alpha \tau} e}{\Delta ; \Xi \vdash \operatorname{box} M \triangleright_{\tau} \Lambda \alpha: \star \rightarrow \star . e} \\
& \overline{\Delta ; \Xi \vdash\langle\rangle \triangleright_{\tau}\langle\rangle[\tau]} \\
& \frac{\Sigma(c)=B \rightarrow b \quad \Delta \vdash B \triangleright_{\tau} \tau_{B}}{\Delta ; \Xi \vdash c \triangleright_{\tau} \lambda x: \tau_{B} \cdot \operatorname{roll}[\tau]\left(\mathbf{i n j}_{\mathcal{L}(c)} x \operatorname{of}\left(\operatorname{Rec} \Sigma^{*} \tau\right)\langle\Sigma\rangle\right)} \\
& \frac{\Delta ; \Xi \vdash M \triangleright_{\tau} e \quad \Delta \vdash A_{1} \triangleright_{\tau} \tau_{1}}{\Delta ; \Xi \vdash \lambda x: A_{1} \cdot M \triangleright_{\tau} \lambda x: \tau_{1} . e} \\
& \frac{\Delta ; \Xi \vdash M_{1} \triangleright_{\tau} e_{1} \quad \Delta ; \Xi \vdash M_{2} \triangleright_{\tau} e_{2}}{\Delta ; \Xi \vdash M_{1} M_{2} \triangleright_{\tau} e_{1} e_{2}} \\
& \Delta \vdash \square A_{1} \triangleright_{\tau} \tau_{1}
\end{aligned}
$$

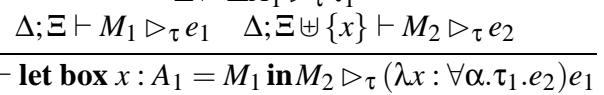

$$
\begin{aligned}
& \frac{\Delta ; \Xi \vdash M_{1} \triangleright_{\tau} e_{1} \quad \Delta ; \Xi \vdash M_{2} \triangleright_{\tau} e_{2}}{\Delta ; \Xi \vdash\left\langle M_{1}, M_{2}\right\rangle \triangleright_{\tau}\left\langle e_{1}, e_{2}\right\rangle} \quad \frac{\Delta ; \Xi \vdash M \triangleright_{\tau} e}{\Delta ; \Xi \vdash \text { fst } M \triangleright_{\tau} \text { fst } e} \\
& \frac{\Delta ; \Xi \vdash M \triangleright_{\tau} e}{\Delta ; \Xi \vdash \operatorname{snd} M \triangleright_{\tau} \mathbf{s n d} e} \\
& \frac{\Delta \vdash A \triangleright_{\tau} \tau_{A} \quad \Delta ; \Xi \vdash \Theta \triangleright_{\tau}^{\tau_{A}} e_{\Theta} \quad \Delta ; \Xi \vdash M \triangleright_{\tau} e_{M}}{\Delta ; \Xi \vdash \operatorname{iter}[\square B, A][\Theta] M \triangleright_{\tau} \operatorname{iter}\left\{B^{*}\right\}[\tau]\left[\tau_{A}\right] e_{\Theta} e_{M}} \\
& \frac{\forall c_{i} \in \operatorname{dom}(\Theta) \quad \Delta ; \Xi \vdash \Theta\left(c_{i}\right) \triangleright_{\tau} e_{i}}{\Delta ; \Xi \vdash \Theta \triangleright{ }_{\tau}^{\tau_{A}} \lambda x: \Sigma^{*} \tau_{A} . \operatorname{case} x \mathbf{o f ~ i n j}_{\mathcal{L}\left(c_{1}\right)} y_{1} \operatorname{in}\left(e_{1} y_{1}\right)} \\
& \operatorname{inj}_{\mathcal{L}\left(c_{n}\right)} y_{n} \text { in }\left(e_{n} y_{n}\right)
\end{aligned}
$$

\section{B.5 Replacements}

